\title{
Partial Hamiltonian reduction of relativistic extended objects in light-cone gauge
}

\author{
Jonas de Woul ${ }^{1, *}$, Jens Hoppe ${ }^{2, \dagger}$, And Douglas Lundholm ${ }^{2, \ddagger}$ \\ ${ }^{1}$ Department of Theoretical Physics, Royal Institute of Technology (KTH) \\ SE-106 91 Stockholm, Sweden \\ ${ }^{2}$ Department of Mathematics, Royal Institute of Technology (KTH) \\ SE-100 44 Stockholm, Sweden
}

May 24, 2022

\begin{abstract}
The elimination of the non-transversal field in the standard light-cone formulation of higher-dimensional extended objects is formulated as a Hamiltonian reduction.
\end{abstract}

\section{Introduction}

While a certain, partially gauge fixed, light-cone formulation of higher dimensional extended objects has, for quite some time [1, been known to yield a polynomial Hamiltonian (density), with the resulting field equations easily checkable by comparing with the corresponding (gauge-fixed) Lagrangian equation of motions, the final step, the elimination of the dynamical longitudinal field and its canonically conjugate momentum (up to their overall integrals) remained — including most string-theory reviews — somewhat unclear concerning the reduction of the Poisson-structure (despite of [2], in which the string case is treated, and [3], in which a Hamiltonian formulation of the supermembrane is given, - as well as [4]). As that reduction to (almost) purely transversal degrees of freedom recently also turned out to be related to generalisations of the Witt-Virasoro algebra, and a novel dynamical symmetry [5, 6, 7, 8], it seems a good idea to give a detailed account of the Hamiltonian reduction (cp. Theorem 4.3) corresponding to the elimination of the longitudinal field.

\footnotetext{
* jodw02@kth.se

$\dagger$ hoppe@math.kth.se

$\ddagger$ dogge@math.kth.se
} 


\section{Preliminaries}

In this section, we recall the Hamiltonian formulation of the extremal volume problem for relativistic extended objects in light-cone gauge.

Consider a $M+1$-dimensional submanifold $\mathcal{M}$ in $D$-dimensional Minkowski space with metric $\eta$ of signature $(+,-, \ldots,-)$. Requiring that the Dirac-Nambu-Goto action (volume functional)

$$
\begin{gathered}
S:=\int \mathcal{L} d \varphi^{0} d^{M} \varphi \\
\mathcal{L}:=-\sqrt{\mathcal{G}}, \quad \mathcal{G}:=\left|\operatorname{det}\left(\mathcal{G}_{\alpha \beta}\right)\right|, \quad \mathcal{G}_{\alpha \beta}:=\partial_{\alpha} x^{\mu} \partial_{\beta} x^{\nu} \eta_{\mu \nu}
\end{gathered}
$$

is stationary under variations of the embedding functions $x^{\mu}, \mu=0,1, \ldots D-1$, gives the equations of motion

$$
\frac{1}{\sqrt{\mathcal{G}}} \partial_{\alpha}\left(\sqrt{\mathcal{G}} \mathcal{G}^{\alpha \beta} \partial_{\beta} x^{\mu}\right)=0
$$

where $\varphi^{0}, \varphi^{1}, \ldots, \varphi^{M}$, collectively denoted by $\left(\varphi^{0}, \varphi\right)$, are local coordinates on $\mathcal{M}$, and $\partial_{\alpha}:=\frac{\partial}{\partial \varphi^{\alpha}}$.

For a Hamiltonian formulation, one introduces canonical momenta

$$
p_{\mu}:=\frac{\partial \mathcal{L}}{\partial \dot{x}^{\mu}}, \quad \mu=0, \ldots, D-1
$$

satisfying the dynamical Poisson bracket:2 (all other brackets zero)

$$
\left\{x^{\mu}(\varphi), p_{\nu}(\tilde{\varphi})\right\}_{P B}=\delta_{\nu}^{\mu} \delta(\varphi, \tilde{\varphi}) .
$$

Because of the general reparameterisation invariance of the action (1), the definition (44) leads to first class primary constraints 3 (see also [10])

$$
\begin{aligned}
& C_{0}:=\frac{1}{2}\left(p_{\mu} p^{\mu}-g\right) \approx 0, \\
& C_{a}:=p_{\mu} \partial_{a} x^{\mu} \approx 0, \quad a=1, \ldots, M,
\end{aligned}
$$

with

$$
g:=\left|\operatorname{det}\left(\left.\mathcal{G}_{a b}\right|_{a, b=1, \ldots, M}\right)\right| .
$$

After the partial gauge fixing

$$
\Phi_{0}:=\frac{x^{0}+x^{D-1}}{2}-\varphi^{0} \approx 0
$$

the constraint $C_{0}$ becomes second class,

$$
\left\{\Phi_{0}(\varphi), C_{0}(\tilde{\varphi})\right\}_{P B}=\pi(\varphi) \delta(\varphi, \tilde{\varphi}),
$$

\footnotetext{
${ }^{1}$ Derivatives with respect to time $\varphi^{0}$ are written $\dot{f}:=\partial_{0} f$.

${ }^{2}$ The equal time coordinate $\varphi^{0}$ is suppressed here and in the following.

${ }^{3}$ See for example [2, 9] for an explanation of the terminology.
} 
with the canonical momenta conjugate to $\zeta:=x^{0}-x^{D-1}$ defined as

$$
\pi:=\frac{\partial \mathcal{L}}{\partial \dot{\zeta}}
$$

while the constraints $C_{a}, a=1, \ldots, M$, remain first class. Following [9, one introduces a corresponding "Dirac bracket" (assuming $\pi \neq 0$ )

$$
\begin{aligned}
\left\{F_{1}, F_{2}\right\}_{D B}:= & \left\{F_{1}, F_{2}\right\}_{P B} \\
& +\int_{\Sigma}\left(\left\{F_{1}, \Phi_{0}(\varphi)\right\}_{P B} \frac{1}{\pi(\varphi)}\left\{C_{0}(\varphi), F_{2}\right\}_{P B}-\left(C_{0} \leftrightarrow \Phi_{0}\right)\right) d^{M} \varphi
\end{aligned}
$$

where $\Sigma$ parametrises the $M$-dimensional extended object. It follows that

$$
\begin{aligned}
\left\{x^{k}(\varphi), p_{l}(\tilde{\varphi})\right\}_{D B} & =\delta_{l}^{k} \delta(\varphi, \tilde{\varphi}), \quad k, l=1, \ldots, D-2 \\
\{\zeta(\varphi), \pi(\tilde{\varphi})\}_{D B} & =\delta(\varphi, \tilde{\varphi}) .
\end{aligned}
$$

After replacing all Poisson brackets with the Dirac brackets (12), the constraints $C_{0}$ and $\Phi_{0}$ can be set strongly to zero. With no risk of confusion, we can drop the subscript ${ }_{D B}$ in (12) and from now on refer to

$$
\left\{F_{1}, F_{2}\right\}:=\int_{\Sigma}\left(\frac{\delta F_{1}}{\delta x^{k}(\varphi)} \frac{\delta F_{2}}{\delta p_{k}(\varphi)}+\frac{\delta F_{1}}{\delta \zeta(\varphi)} \frac{\delta F_{2}}{\delta \pi(\varphi)}-\left(F_{1} \leftrightarrow F_{2}\right)\right) d^{M} \varphi
$$

as the dynamical Poisson bracket (thus replacing (5) ).

The action (1) can then be written

$$
S=\int\left(\pi \dot{\zeta}+\vec{p} \cdot \dot{\vec{x}}-\frac{\vec{p}^{2}+g}{-2 \pi}\right) d \varphi^{0} d^{M} \varphi
$$

with the corresponding Hamiltonian (see also [4])

$$
H[\vec{x}, \zeta ; \vec{p}, \pi]:=\int_{\Sigma} \frac{\vec{p}^{2}+g}{-2 \pi} d^{M} \varphi
$$

where

$$
g=\operatorname{det}\left(g_{a b}\right), \quad g_{a b}:=\partial_{a} \vec{x} \cdot \partial_{b} \vec{x}, \quad a, b=1, \ldots, M,
$$

and the Euclidean vectors $\vec{x}$ and $\vec{p}$ having components $x^{k}$ and $p_{k}, k=1, \ldots, D-2$, respectively. The remaining first class constraints become

$$
C_{a}=\pi \partial_{a} \zeta+\vec{p} \cdot \partial_{a} \vec{x} \approx 0, \quad a=1, \ldots, M
$$

which, by eliminating $\zeta$, locally give the integrability conditions

$$
\partial_{a}\left(\frac{\vec{p}}{\pi}\right) \cdot \partial_{b} \vec{x}-\partial_{b}\left(\frac{\vec{p}}{\pi}\right) \cdot \partial_{a} \vec{x} \approx 0, \quad 1 \leq a<b \leq M .
$$


Since the Hamiltonian (15) does not depend on the phase-space field $\zeta$, its conjugate momentum is conserved, $\dot{\pi}(\varphi)=0$. We can therefore write for the solution to this equation of motion

$$
\pi(\varphi)=-\eta \rho(\varphi)
$$

with a discrete dynamical variable $\eta$ satisfying $\dot{\eta}=0$, and a non-dynamical positive density $\rho$ normalized such that

$$
\int_{\Sigma} \rho(\varphi) d^{M} \varphi=1
$$

Furthermore, (17) allows us to solve for $\zeta$ (in terms of $\vec{x}, \vec{p}$ and $\eta$ ) up to the zero mode

$$
\zeta_{0}:=\int_{\Sigma} \rho(\varphi) \zeta(\varphi) d^{M} \varphi
$$

The plan of this note is to perform a further partial Hamiltonian reduction such that the canonical fields $\zeta$ and $\pi$ can be discarded in favour of the discrete zero mode variables $\zeta_{0}$ and $\eta$, the latter satisfying

$$
\left\{\zeta_{0}, \eta\right\}=-1
$$

and with the field variables $\vec{x}$ and $\vec{p}$ then constrained (locally) by (cf. (18))

$$
\Phi_{a b}:=\partial_{a}\left(\frac{\vec{p}}{\rho}\right) \cdot \partial_{b} \vec{x}-\partial_{b}\left(\frac{\vec{p}}{\rho}\right) \cdot \partial_{a} \vec{x} \approx 0, \quad 1 \leq a<b \leq M .
$$

\section{Constraints}

In this section, we perform a partial gauge fixing corresponding to (19), and introduce the primary constraint that becomes second class as a result of this gauge fixing.

In the following, the domain of integration is always $\Sigma$. Introduce a metric $\rho_{a b}$ on $\Sigma$ such that $\sqrt{\operatorname{det}\left(\rho_{a b}\right)}=\rho$ and denote the non-constant eigenfunctions, respectively eigenvalues, of the corresponding Laplacian on $\Sigma$ by $Y_{\alpha}$ and $-\mu_{\alpha}$ respectively, i.e.

$$
-\Delta Y_{\alpha}=-\frac{1}{\rho} \partial_{a}\left(\rho \partial^{a}\right) Y_{\alpha}=\mu_{\alpha} Y_{\alpha}, \quad \alpha=1,2, \ldots
$$

with $\partial^{a}:=\rho^{a b} \partial_{b}$. The eigenfunctions are normalized such that

$$
\int Y_{\alpha}(\varphi) Y_{\beta}(\varphi) \rho(\varphi) d^{M} \varphi=\delta_{\alpha, \beta}
$$

resulting in

$$
\rho(\varphi)+\sum_{\alpha=1}^{\infty} \rho(\varphi) Y_{\alpha}(\varphi) Y_{\alpha}(\tilde{\varphi})=\delta(\varphi, \tilde{\varphi})
$$

Defining

$$
G(\varphi, \tilde{\varphi}):=-\sum_{\alpha=1}^{\infty} \frac{1}{\mu_{\alpha}} Y_{\alpha}(\varphi) Y_{\alpha}(\tilde{\varphi})
$$


one has 11

$$
\Delta_{\tilde{\varphi}} G(\varphi, \tilde{\varphi})=\frac{\delta(\varphi, \tilde{\varphi})}{\rho(\varphi)}-1
$$

and

$$
\int G(\varphi, \tilde{\varphi}) \rho(\varphi) d^{M} \varphi=-\sum_{\alpha=1}^{\infty} \frac{1}{\mu_{\alpha}} Y_{\alpha}(\tilde{\varphi}) \delta_{0, \alpha}=0 .
$$

Definition 3.1. Introduce the constraint functions

$$
\begin{aligned}
& \phi_{1}(\varphi):=\zeta(\varphi)-\int \zeta(\tilde{\varphi}) \rho(\tilde{\varphi}) d^{M} \tilde{\varphi}+\Phi(\varphi), \\
& \phi_{2}(\varphi):=\pi(\varphi)-\rho(\varphi) \int \pi(\tilde{\varphi}) d^{M} \tilde{\varphi}
\end{aligned}
$$

with

$$
\Phi(\varphi):=\int G^{a}(\varphi, \tilde{\varphi}) \frac{\vec{p}(\tilde{\varphi}) \cdot \tilde{\partial}_{a} \vec{x}(\tilde{\varphi})}{\pi(\tilde{\varphi})} \rho(\tilde{\varphi}) d^{M} \tilde{\varphi}
$$

and (introduced in [1] $) G^{a}(\varphi, \tilde{\varphi}):=-\tilde{\partial}^{a} G(\varphi, \tilde{\varphi})$.

Using the primary constraints (17) in (31), integrating by parts, and applying (28) gives

$$
\begin{aligned}
\phi_{1}(\varphi) & \approx \zeta(\varphi)-\int \zeta(\tilde{\varphi}) \rho(\tilde{\varphi}) d^{M} \tilde{\varphi}+\int G^{a}(\varphi, \tilde{\varphi})\left(-\tilde{\partial}_{a} \zeta(\tilde{\varphi})\right) \rho(\tilde{\varphi}) d^{M} \tilde{\varphi} \\
& =\zeta(\varphi)-\int \zeta(\tilde{\varphi}) \rho(\tilde{\varphi}) d^{M} \tilde{\varphi}-\int \zeta(\tilde{\varphi}) \rho(\tilde{\varphi}) \Delta_{\tilde{\varphi}} G(\varphi, \tilde{\varphi}) d^{M} \tilde{\varphi}=0,
\end{aligned}
$$

which means that $\phi_{1}$ is a primary constraint. Indeed, one easily checks that

$$
\phi_{1}(\varphi)=\int G^{a}(\varphi, \tilde{\varphi}) \frac{1}{\pi(\tilde{\varphi})} C_{a}(\tilde{\varphi}) \rho(\tilde{\varphi}) d^{M} \tilde{\varphi}
$$

with $C_{a}$ given in (17). We now impose

$$
\phi_{2}(\varphi) \approx 0
$$

as an additional gauge constraint. Using this, one can solve for $\pi$ in terms of the discrete variable $\eta=-\int \pi d^{M} \varphi$ (cf. (19) $)$ ) on the constraint surface.

Lemma 3.2. The constraints (30) satisfy the dynamical Poisson brackets

$$
\begin{aligned}
& \left\{\phi_{1}(\varphi), \phi_{1}(\tilde{\varphi})\right\} \approx 0, \\
& \left\{\phi_{2}(\varphi), \phi_{2}(\tilde{\varphi})\right\}=0,
\end{aligned}
$$

and

$$
\left\{\phi_{1}(\varphi), \phi_{2}(\tilde{\varphi})\right\}=\delta(\varphi, \tilde{\varphi})-\rho(\tilde{\varphi})
$$


Proof. The following variational derivatives of (31) will be needed:

$$
\begin{aligned}
\frac{\delta \Phi(\varphi)}{\delta \zeta(\tilde{\varphi})} & =0 \\
\frac{\delta \Phi(\varphi)}{\delta \pi(\tilde{\varphi})} & =-G^{a}(\varphi, \tilde{\varphi}) \frac{\vec{p}(\tilde{\varphi}) \cdot \tilde{\partial}_{a} \vec{x}(\tilde{\varphi})}{\pi(\tilde{\varphi})^{2}} \rho(\tilde{\varphi}), \\
\frac{\delta \Phi(\varphi)}{\delta x^{k}(\tilde{\varphi})} & =-\tilde{\partial}_{a}\left(G^{a}(\varphi, \tilde{\varphi}) \frac{p_{k}(\tilde{\varphi})}{\pi(\tilde{\varphi})} \rho(\tilde{\varphi})\right), \\
\frac{\delta \Phi(\varphi)}{\delta p_{k}(\tilde{\varphi})} & =G^{a}(\varphi, \tilde{\varphi}) \frac{\tilde{\partial}_{a} x^{k}(\tilde{\varphi})}{\pi(\tilde{\varphi})} \rho(\tilde{\varphi}) .
\end{aligned}
$$

Inserting (30) on the left hand side of (35) yields

$$
\left\{\phi_{1}(\varphi), \phi_{1}(\tilde{\varphi})\right\}=\frac{\delta \Phi(\tilde{\varphi})}{\delta \pi(\varphi)}-\frac{\delta \Phi(\varphi)}{\delta \pi(\tilde{\varphi})}+\int\left(\frac{\delta \Phi(\varphi)}{\delta \pi(\hat{\varphi})}-\frac{\delta \Phi(\tilde{\varphi})}{\delta \pi(\hat{\varphi})}\right) \rho(\hat{\varphi}) d^{M} \hat{\varphi}+\{\Phi(\varphi), \Phi(\tilde{\varphi})\}
$$

where we have used that

$$
\{\zeta(\varphi), \Phi(\tilde{\varphi})\}=\frac{\delta \Phi(\tilde{\varphi})}{\delta \pi(\varphi)} .
$$

Using the definition of the Poisson bracket, the variational derivatives (38), and integrating by parts gives

$$
\begin{aligned}
\{\Phi(\varphi), \Phi(\tilde{\varphi})\} & =\int \frac{\vec{p}(\hat{\varphi}) \cdot \hat{\partial}_{b} \vec{x}(\hat{\varphi})}{\pi(\hat{\varphi})^{2}} \rho(\hat{\varphi})^{2}\left(G^{b}(\tilde{\varphi}, \hat{\varphi}) \Delta_{\hat{\varphi}} G(\varphi, \hat{\varphi})-G^{b}(\varphi, \hat{\varphi}) \Delta_{\hat{\varphi}} G(\tilde{\varphi}, \hat{\varphi})\right) d^{M} \hat{\varphi} \\
& -\int G^{a}(\varphi, \hat{\varphi}) G^{b}(\tilde{\varphi}, \hat{\varphi}) \frac{\rho(\hat{\varphi})^{2}}{\pi(\hat{\varphi})}\left(\hat{\partial}_{a}\left(\frac{\vec{p}}{\pi}\right) \cdot \hat{\partial}_{b} \vec{x}-\hat{\partial}_{b}\left(\frac{\vec{p}}{\pi}\right) \cdot \hat{\partial}_{a} \vec{x}\right) d^{M} \hat{\varphi} .
\end{aligned}
$$

Applying (18) to the expression within parenthesis in the last line above, together with the use of equations (28) and (38), leads to

$$
\{\Phi(\varphi), \Phi(\tilde{\varphi})\} \approx-\frac{\delta \Phi(\tilde{\varphi})}{\delta \pi(\varphi)}+\frac{\delta \Phi(\varphi)}{\delta \pi(\tilde{\varphi})}-\int\left(\frac{\delta \Phi(\varphi)}{\delta \pi(\hat{\varphi})}-\frac{\delta \Phi(\tilde{\varphi})}{\delta \pi(\hat{\varphi})}\right) \rho(\hat{\varphi}) d^{M} \hat{\varphi}
$$

which proves (35). The Poisson bracket (36) is trivial since $\phi_{2}(\varphi)$ only involves the field $\pi(\varphi)$. Finally, (37) is easily proven using (15), (20), and by noting that

$$
\{\Phi(\varphi), \pi(\tilde{\varphi})\}=0
$$

since $\Phi$ does not contain $\zeta$.

\section{Dirac bracket}

In this section, we define a Dirac bracket that turns the second class constraints (30) effectively first class. To this end, the canonical procedure would be to set

$$
C_{a b}(\varphi, \tilde{\varphi}):=\left\{\phi_{a}(\varphi), \phi_{b}(\tilde{\varphi})\right\}, \quad a, b=1,2
$$


and then define the Dirac bracket by

$$
\left\{F_{1}, F_{2}\right\}^{*}=\left\{F_{1}, F_{2}\right\}-\sum_{a, b=1,2} \int\left\{F_{1}, \phi_{a}(\varphi)\right\}\left(C^{-1}\right)_{a b}(\varphi, \tilde{\varphi})\left\{\phi_{b}(\tilde{\varphi}), F_{2}\right\} d^{M} \varphi d^{M} \tilde{\varphi} .
$$

However, as it stands, this expression is not well defined since (39) is not invertible. More specifically, by equation (37) in Lemma 3.2, one finds that

$$
\chi(\varphi, \tilde{\varphi}):=C_{12}(\varphi, \tilde{\varphi})
$$

defines a projection onto the space of zero-mean real-valued functions on $\Sigma$ :

$$
[\chi f](\varphi):=\int \chi(\varphi, \tilde{\varphi}) f(\tilde{\varphi}) d^{M} \tilde{\varphi}=f(\varphi)-\bar{f}
$$

with the mean of $f$ defined by

$$
\bar{f}:=\int f(\varphi) \rho(\varphi) d^{M} \varphi
$$

It follows that $\operatorname{ker}(\chi)=\{f: \Sigma \rightarrow \mathbb{R} \mid f$ constant $\}$ is non-trivial, and (39) is indeed noninvertible. Fortunately, it turns out that the bracket obtained by restricting (41) to $\mathrm{im}(\chi)=$ $\{f: \Sigma \rightarrow \mathbb{R} \mid \bar{f}=0\}$, i.e. $\left.\chi\right|_{\operatorname{im}(\chi)}=\delta(\varphi, \tilde{\varphi})$, will be sufficient for our purposes.

Definition 4.1. Define

$$
\left\{F_{1}, F_{2}\right\}^{*}:=\left\{F_{1}, F_{2}\right\}+\int\left(\left\{F_{1}, \phi_{1}(\varphi)\right\}\left\{\phi_{2}(\varphi), F_{2}\right\}-\left\{F_{1}, \phi_{2}(\varphi)\right\}\left\{\phi_{1}(\varphi), F_{2}\right\}\right) d^{M} \varphi .
$$

This bracket has the desired properties, as seen by the following:

Lemma 4.2. The bracket (44) satisfies

$$
\left\{\phi_{k}, \phi_{l}\right\}^{*} \approx 0, \quad k, l=1,2 .
$$

Furthermore, for arbitrary functions of $\vec{x}, \zeta_{0}, \vec{p}$, and $\pi$ (in particular $\eta$ ), it holds that

$$
\begin{gathered}
\left\{F_{1}, F_{2}\right\}^{*}=-\left\{F_{2}, F_{1}\right\}^{*}, \\
\left\{F_{1}, F_{2} F_{3}\right\}^{*}=\left\{F_{1}, F_{2}\right\}^{*} F_{3}+F_{2}\left\{F_{1}, F_{3}\right\}^{*}, \\
\left\{\left\{F_{1}, F_{2}\right\}^{*}, F_{3}\right\}^{*}+\left\{\left\{F_{2}, F_{3}\right\}^{*}, F_{1}\right\}^{*}+\left\{\left\{F_{3}, F_{1}\right\}^{*}, F_{2}\right\}^{*}=0,
\end{gathered}
$$

and

$$
\left\{F, \phi_{k}\right\}^{*}=0, \quad k=1,2
$$

Proof. The brackets (45) follow immediately using Lemma 3.2 and the projection property of $\chi$, while relations (46) and (47) are trivial. The Jacobi identity is proven along the same lines as the original proof by Dirac [12]. To simplify the algebra, we first write (44) symbolically as

$$
\begin{aligned}
\left\{F_{1}, F_{2}\right\}^{*} & =\left\{F_{1}, F_{2}\right\}+\iint\left\{F_{1}, \phi_{a}(\varphi)\right\} M_{a b}(\varphi, \tilde{\varphi})\left\{\phi_{b}(\tilde{\varphi}), F_{2}\right\} d^{M} \varphi d^{M} \tilde{\varphi} \\
& =\left\{F_{1}, F_{2}\right\}+\left\{F_{1}, \phi_{a}\right\} M_{a b}\left\{\phi_{b}, F_{2}\right\}
\end{aligned}
$$


with $M_{11}=M_{22}=0$ and $M_{12}(\varphi, \tilde{\varphi})=-M_{21}(\varphi, \tilde{\varphi})=\delta(\varphi, \tilde{\varphi})$. Let $\mathcal{S}$ denote cyclic permutation of $1,2,3$, and summation of the result, such that

$$
\mathcal{S}\left(\left\{\left\{F_{1}, F_{2}\right\}^{*}, F_{3}\right\}^{*}\right)=\left\{\left\{F_{1}, F_{2}\right\}^{*}, F_{3}\right\}^{*}+\left\{\left\{F_{2}, F_{3}\right\}^{*}, F_{1}\right\}^{*}+\left\{\left\{F_{3}, F_{1}\right\}^{*}, F_{2}\right\}^{*} .
$$

Then

$$
\begin{aligned}
\mathcal{S}\left(\left\{\left\{F_{1}, F_{2}\right\}^{*}, F_{3}\right\}^{*}\right) & =\mathcal{S}\left(\left\{\left\{F_{1}, F_{2}\right\}, F_{3}\right\}\right) \\
& +M_{r s} \mathcal{S}\left(\left\{F_{1}, \phi_{r}\right\}\left(\left\{\left\{\phi_{s}, F_{2}\right\}, F_{3}\right\}+\left\{\left\{F_{3}, \phi_{s}\right\}, F_{2}\right\}+\left\{\left\{F_{2}, F_{3}\right\}, \phi_{s}\right\}\right)\right) \\
& +M_{r s} M_{t u} \mathcal{S}\left(\left\{\left\{F_{1}, \phi_{r}\right\}\left\{\phi_{s}, F_{2}\right\}, \phi_{t}\right\}\left\{\phi_{u}, F_{3}\right\}\right)
\end{aligned}
$$

The first two lines are zero by the Jacobi identity for the Poisson bracket. Renaming dummy summation indices and again using the Jacobi identity, the last line can be rewritten as

$$
\begin{aligned}
\mathcal{S}\left(\left\{\left\{F_{1}, F_{2}\right\}^{*}, F_{3}\right\}^{*}\right) & =M_{r s} M_{t u} \mathcal{S}\left(\left\{\phi_{s}, F_{2}\right\}\left\{\phi_{u}, F_{3}\right\}\left(\left\{\left\{F_{1}, \phi_{r}\right\}, \phi_{t}\right\}+\left\{\left\{\phi_{t}, F_{1}\right\}, \phi_{r}\right\}\right)\right) \\
& =-M_{r s} M_{t u} \mathcal{S}\left(\left\{\phi_{s}, F_{2}\right\}\left\{\phi_{u}, F_{3}\right\}\left\{\left\{\phi_{r}, \phi_{t}\right\}, F_{1}\right\}\right)
\end{aligned}
$$

By Lemma 3.2, this simplifies to

$$
\mathcal{S}\left(\left\{\left\{F_{1}, F_{2}\right\}^{*}, F_{3}\right\}^{*}\right)=-M_{12} M_{12} \mathcal{S}\left(\left\{\phi_{2}, F_{2}\right\}\left\{\phi_{2}, F_{3}\right\}\left\{\left\{\phi_{1}, \phi_{1}\right\}, F_{1}\right\}\right)
$$

when summing over indices. But this is zero since

$$
\left\{\zeta_{0}, \phi_{2}(\varphi)\right\}=0 \Rightarrow\left\{F, \phi_{2}(\varphi)\right\}=0
$$

for functions $F$ of $\vec{x}, \zeta_{0}, \vec{p}$, and $\pi$. To prove (49), we first note that

$$
\begin{aligned}
\int\left\{F, \phi_{1}(\varphi)\right\} \rho(\varphi) d^{M} \varphi & =\int\left(\frac{\delta F}{\delta x^{k}(\tilde{\varphi})} \frac{\delta \Phi(\varphi)}{\delta p_{k}(\tilde{\varphi})}+\frac{\delta F}{\delta \zeta(\tilde{\varphi})} \frac{\delta \Phi(\varphi)}{\delta \pi(\tilde{\varphi})}\right. \\
& \left.-\frac{\delta \Phi(\varphi)}{\delta x^{k}(\tilde{\varphi})} \frac{\delta F}{\delta p_{k}(\tilde{\varphi})}-\frac{\delta \phi_{1}(\varphi)}{\delta \zeta(\tilde{\varphi})} \frac{\delta F}{\delta \pi(\tilde{\varphi})}\right) \rho(\varphi) d^{M} \varphi d^{M} \tilde{\varphi} \\
& =-\int \frac{\delta \phi_{1}(\varphi)}{\delta \zeta(\tilde{\varphi})} \frac{\delta F}{\delta \pi(\tilde{\varphi})} \rho(\varphi) d^{M} \varphi d^{M} \tilde{\varphi} \\
& =-\int(\delta(\varphi, \tilde{\varphi})-\rho(\tilde{\varphi})) \frac{\delta F}{\delta \pi(\tilde{\varphi})} \rho(\varphi) d^{M} \varphi d^{M} \tilde{\varphi}=0
\end{aligned}
$$

where, in the second equality, we have used that variations of $\Phi$ (38) depend explicitly on $G^{a}(\varphi, \tilde{\varphi})$. Analogous to (29), all these terms vanish when one integrates with respect to $\varphi$ above. It follows that for an arbitrary function $F$ of $\vec{x}, \zeta_{0}, \vec{p}$, and $\pi$,

$$
\begin{aligned}
& \left\{F, \phi_{1}(\varphi)\right\}^{*}=\int\left(\left\{F, \phi_{1}(\hat{\varphi})\right\} \rho(\hat{\varphi})-\left\{F, \phi_{2}(\hat{\varphi})\right\}\left\{\phi_{1}(\hat{\varphi}), \phi_{1}(\varphi)\right\}\right) d^{M} \hat{\varphi}=0, \\
& \left\{F, \phi_{2}(\varphi)\right\}^{*}=\rho(\varphi) \int\left\{F, \phi_{2}(\hat{\varphi})\right\} d^{M} \hat{\varphi}=0 .
\end{aligned}
$$

The Hamiltonian reduction of the degrees of freedom is completed by: 
Theorem 4.3. The phase space variables $\vec{x}, \zeta_{0}, \vec{p}$, and $\eta$ satisfy

$$
\left\{\zeta_{0}, \eta\right\}^{*}=-1, \quad\left\{x^{k}(\varphi), p_{l}(\tilde{\varphi})\right\}^{*}=\delta_{l}^{k} \delta(\varphi, \tilde{\varphi}), \quad k, l=1, \ldots D-2,
$$

with all other brackets zero, and the reduced Hamiltonian is

$$
H\left[\vec{x}, \zeta_{0} ; \vec{p}, \eta\right]=\frac{1}{2 \eta} \int_{\Sigma} \frac{\vec{p}^{2}+g}{\rho} d^{M} \varphi .
$$

Proof. We apply Definition 4.1 (suppressing the arguments of the field variables whenever there is no risk of confusion). Since $\left\{\phi_{2}, x^{k}\right\}=\left\{\phi_{2}, p_{l}\right\}=0$, the Dirac brackets between the $\vec{x}$ and $\vec{p}$ coincide with the corresponding dynamical Poisson brackets $\left(\left\{x^{k}, x^{l}\right\}^{*}=\left\{x^{k}, x^{l}\right\}\right.$, etc.). Furthermore,

$$
\begin{aligned}
\left\{\zeta_{0}, \eta\right\}^{*} & =\left\{\zeta_{0}, \eta\right\}+\int\left(\left\{\zeta_{0}, \phi_{1}(\varphi)\right\}\left\{\phi_{2}(\varphi), \eta\right\}-\left\{\zeta_{0}, \phi_{2}(\varphi)\right\}\left\{\phi_{1}(\varphi), \eta\right\}\right) d^{M} \varphi \\
\left\{\zeta_{0}, x^{k}\right\}^{*} & =\left\{\zeta_{0}, x^{k}\right\}-\int\left\{\zeta_{0}, \phi_{2}(\varphi)\right\}\left\{\phi_{1}(\varphi), x^{k}\right\} d^{M} \varphi \\
\left\{\zeta_{0}, p_{k}\right\}^{*} & =\left\{\zeta_{0}, p_{k}\right\}-\int\left\{\zeta_{0}, \phi_{2}(\varphi)\right\}\left\{\phi_{1}(\varphi), p_{k}\right\} d^{M} \varphi \\
\left\{\eta, x^{k}\right\}^{*} & =\left\{\eta, x^{k}\right\}-\int\left\{\eta, \phi_{2}(\varphi)\right\}\left\{\phi_{1}(\varphi), x^{k}\right\} d^{M} \varphi \\
\left\{\eta, p_{k}\right\}^{*} & =\left\{\eta, p_{k}\right\}-\int\left\{\eta, \phi_{2}(\varphi)\right\}\left\{\phi_{1}(\varphi), p_{k}\right\} d^{M} \varphi
\end{aligned}
$$

The result now follows by using (5) $(22)$, and the fact that the dynamical Poisson brackets

$$
\left\{\zeta_{0}, \phi_{1}\right\}, \quad\left\{\zeta_{0}, \phi_{2}\right\}, \quad\left\{\zeta_{0}, x^{k}\right\}, \quad\left\{\zeta_{0}, p_{k}\right\}, \quad\left\{\eta, \phi_{1}\right\}, \quad\left\{\eta, \phi_{2}\right\}, \quad\left\{\eta, x^{k}\right\}, \quad\left\{\eta, p_{k}\right\},
$$

all vanish.

\section{Acknowledgments}

This work was supported by the Göran Gustafsson Foundation and the Knut and Alice Wallenberg Foundation (grant KAW 2005.0098).

\section{A Other notational conventions}

For the convenience of the reader, some relations between our notation and another one often used in the literature are summarised in this appendix. Taking the metric signature $\eta \sim(+,-, \ldots,-)$, we have followed here the convention that light-cone coordinates are defined by

$$
x^{+}:=\frac{1}{2}\left(x^{0}+x^{D-1}\right), \quad x^{-} \equiv \zeta:=x^{0}-x^{D-1},
$$


and the scalar product is given by

$$
a \cdot b=a^{\mu} b_{\mu}=a^{+} b^{-}+a^{-} b^{+}-\vec{a} \cdot \vec{b} .
$$

On the other hand, it is also common in the literature to use reversed metric signature $\tilde{\eta} \sim(-,+, \ldots,+)$, with the convention

$$
\tilde{x}^{ \pm}:=\frac{1}{\sqrt{2}}\left(x^{D-1} \pm x^{0}\right)
$$

and scalar product

$$
\tilde{a} \cdot \tilde{b}=\tilde{a}^{\mu} \tilde{b}_{\mu}=\tilde{a}^{+} \tilde{b}^{-}+\tilde{a}^{-} \tilde{b}^{+}+\tilde{\vec{a}} \cdot \tilde{\vec{b}}=-a \cdot b,
$$

so that a translation between notations is given by

$$
\tilde{x}^{+}=\sqrt{2} x^{+}, \quad \tilde{x}^{-}=-\frac{1}{\sqrt{2}} \zeta, \quad \tilde{\vec{x}}=\vec{x} .
$$

After gauge fixing $\varphi^{0} \stackrel{!}{=} \tilde{x}^{+}$, the conjugate momenta become (the expression for $\tilde{p}^{-}$following from the constraint $C_{0}$ in ([6])

$$
\tilde{p}^{+}:=\frac{\partial \mathcal{L}}{\partial\left(\partial_{0} \tilde{x}^{-}\right)}=-\sqrt{2} \pi, \quad \tilde{p}^{-}=-\frac{\tilde{\vec{p}}^{2}+g}{2 \tilde{p}^{+}}, \quad \tilde{\vec{p}}=\vec{p}
$$

and the light-cone Hamiltonian

$$
\tilde{H}=-\tilde{P}^{-}=\frac{1}{\sqrt{2}} H, \quad \tilde{P}^{-}:=\int_{\Sigma} \tilde{p}^{-} d^{M} \varphi .
$$

The zero mode variables remaining after the reduction of the phase space are

$$
\tilde{X}^{-}:=\int_{\Sigma} \tilde{x}^{-} \rho d^{M} \varphi=-\frac{1}{\sqrt{2}} \zeta_{0}, \quad \tilde{P}^{+}:=\int_{\Sigma} \tilde{p}^{+} d^{M} \varphi=\sqrt{2} \eta,
$$

with $\left\{\tilde{X}^{-}, \tilde{P}^{+}\right\}^{*}=-\left\{\zeta_{0}, \eta\right\}^{*}=1$. The mass-squared of the theory is given by

$$
\mathbb{M}^{2}:=P \cdot P=-\tilde{P} \cdot \tilde{P}=-2 \tilde{P}^{+} \tilde{P}^{-}-\tilde{\vec{P}}^{2}=\int_{\Sigma} \frac{\vec{p}^{2}+g}{\rho} d^{M} \varphi-\left(\int_{\Sigma} \vec{p} d^{M} \varphi\right)^{2} .
$$

\section{References}

[1] J. Hoppe, Quantum Theory of a Massless Relativistic Surface and a Two-dimensional Bound State Problem, Ph.D. Thesis, MIT 1982; http://dspace.mit.edu/handle/1721.1/15717

[2] A. Hanson, T. Regge, C. Teitelboim, Constrained Hamiltonian systems, Accademia Nazionale dei Lincei (1976)

[3] E. Bergshoeff, E. Sezgin, Y. Tanii, Hamiltonian formulation of the supermembrane, Nucl. Phys. B. 298, 187 (1988) 
[4] J. Hoppe, Membranes and Matrix Models, arXiv:hep-th/0206192, IHES/P/02/47

[5] J. Hoppe, Fundamental Structures of M(brane) Theory, arXiv:1003.5189

[6] J. de Woul, J. Hoppe, D. Lundholm and M. Sundin, A dynamical symmetry for supermembranes, arXiv:1004.0266

[7] J. Hoppe, Quantum Reconstruction Algebras, http://urn.kb.se/resolve?urn=urn:nbn:se:kth:diva-13591 (submitted to arXiv May 20 2010)

[8] J. Hoppe, Matrix Models and Lorentz Invariance; manuscript / lectures given at KTH in March 2010; to appear

[9] P. A. M. Dirac, Lectures on Quantum Mechanics, Belfer Graduate School of Science, Yeshiva University, New York (1964)

[10] V. Moncrief, Can one ADM quantize relativistic bosonic strings and membranes?, Gen. Relativ. Gravit. 38, 561 (2006)

[11] J. Goldstone, unpublished notes

[12] P. A. M. Dirac, Generalized Hamiltonian dynamics, Canad. J. Math. 2, 129 (1950) 